\title{
Isolated pancreatic tuberculosis mimicking inoperable pancreatic cancer: A diagnostic challenge resolved by endoscopic ultrasound-guided fine-needle aspiration
}

\author{
Adrien Sportes MD¹, Raimi Kpossou MD¹, Stephanie Bernardin MD²
}

\section{CASE PRESENTATION}

In December 2010, a 52-year-old man was admitted to hospital for febrile cholestatic jaundice. The patient was of Algerian origin and had immigrated to Alsace (France) two years previously. He was a construction worker with no medical or surgical history, nor was there history of contact with tuberculosis. The clinical history began one month previously, with diffuse abdominal pain, weight loss and jaundice.

Physical examination on admission revealed a temperature of $38.5^{\circ} \mathrm{C}$, epigastric abdominal pain associated with scleral jaundice and no lymphadenopathy. The remainder of the physical examination was unremarkable.

The patient's initial laboratory analysis revealed a leukocyte count of $7.07 \times 10^{9} / \mathrm{L}$, a hemoglobin level of $97 \mathrm{~g} / \mathrm{L}$ and a platelet count of $259 \times 10^{9} / \mathrm{L}$. Cholestasis was demonstrated by increased serum levels of gamma-glutamyltransferase ( $336 \mathrm{U} / \mathrm{L}$; normal range $11 \mathrm{U} / \mathrm{L}$ to $82 \mathrm{U} / \mathrm{L})$, alkaline phosphatase (177 U/L; normal range $41 \mathrm{U} / \mathrm{L}$ to $117 \mathrm{U} / \mathrm{L})$ and total bilirubin ( $30 \mu \mathrm{mol} / \mathrm{L}$; normal range $1.7 \mu \mathrm{mol} / \mathrm{L}$ to $21 \mu \mathrm{mol} / \mathrm{L})$, with a direct bilirubin level of $20 \mu \mathrm{mol} / \mathrm{L}$ (normal range $1 \mu \mathrm{mol} / \mathrm{L}$ to $10 \mu \mathrm{mol} / \mathrm{L}$ ) and alanine aminotransferase level of $120 \mathrm{U} / \mathrm{L}$ (normal range $10 \mathrm{U} / \mathrm{L}$ to $49 \mathrm{U} / \mathrm{L}$ ). Acute phase reactants increased, with a C-reactive protein level of $63 \mathrm{mg} / \mathrm{L}$ (normal $<4 \mathrm{mg} / \mathrm{L}$ ). Tests for HIV 1 and 2 , and hepatitis $\mathrm{B}$ and $\mathrm{C}$ were negative.

Computed tomography (CT) showed a heterogeneous mass in the head of the pancreas $3.5 \mathrm{~cm} \times 4.5 \mathrm{~cm}$ in size, causing a compression of the bile duct, with dilation of the intra- and extrahepatic bile ducts. The tumour was in contact with the superior mesenteric vein and artery. In addition, many peripancreatic lymph nodes were present (Figure 1).

Magnetic resonance cholangiopancreatography was performed and revealed dilation of the intra- and extrahepatic bile ducts upstream of a cephalic pancreatic mass, with dilation of the duct of Wirsung (Figure 2).

The initial differential diagnosis was cholangitis secondary to a malignant tumour of the pancreatic head without knowledge of the histological type.

Given the septic context and the presence of a locally advanced tumour (invasion of the superior mesenteric artery and vein), it was decided to perform endoscopic ultrasound (EUS)-guided fine-needle aspiration (FNA) of the mass of the head of the pancreas, along with placement of a metal biliary stent. EUS revealed a large tumour in the head region of the pancreas compressing the bile duct, and many peripancreatic lymph nodes and some ascites (Figure 3).

Cytology demonstrated granuloma with caseous necrosis and the presence of an aspect of diffuse necrosis, the absence of tumour cells and tuberculosis bacterium. The assessment was completed using a CT scan-guided transmural biopsy of the peripancreatic lymphadenopathy. Pathological examination revealed caseous necrosis with the presence of acid-fast bacilli; Ziehl-Neelsen stain staining was positive. Additionally, bacteriological samples returned positive for Mycobacterium tuberculosis with polymerase chain reaction DNA.

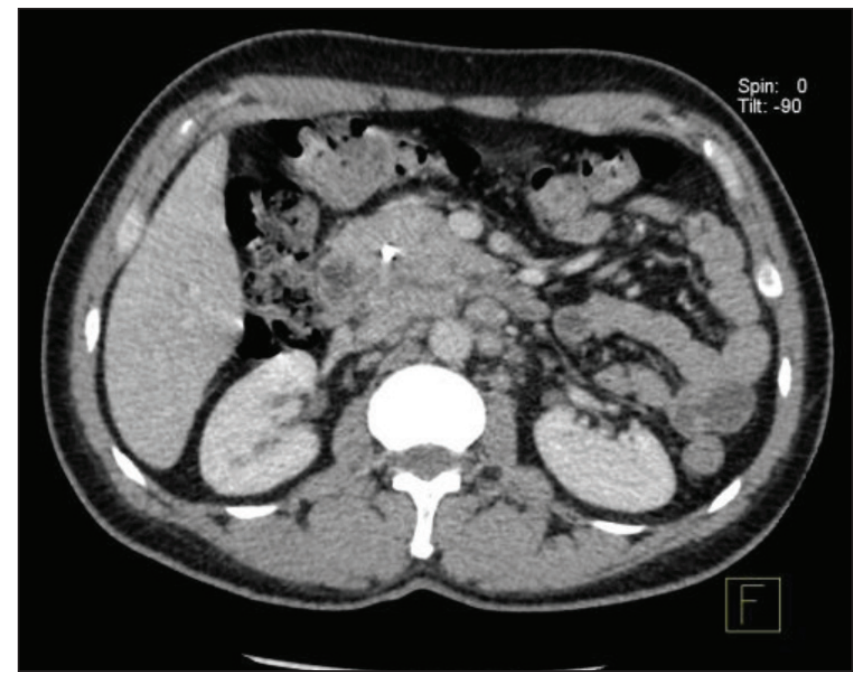

Figure 1) Abdominal computed tomography scan showing a heterogeneous mass in the head of the pancreas

Positron emission CT was used to search for other sites of tuberculosis infection. Examination revealed hyperintense metabolism characterizing the large swelling of the head of the pancreas associated with multiple gastric lymph nodes and perihepatic hilum (Figure 4).

The final diagnosis was isolated pancreatic tuberculosis complicated by cholangitis. Antituberculous therapy was initiated, leading to clinical, biological and radiological improvement (Figure 5). The metal biliary stent was replaced with a plastic stent. It was replaced once again before its final removal in September 2011.

\section{DISCUSSION}

Isolated pancreatic tuberculosis is rare (1), even in endemic countries. In France, two cases have been reported in the literature. Pancreatic tuberculosis is most often associated with miliary tuberculosis $(2,3)$ or immunosuppression by HIV. Our patient had no miliary tuberculosis or HIV infection, or other causes of immunosuppression.

The pathogenesis in this context may be due to hematogenous extension (4). Invasion of the pancreas by tubercle bacilli disseminated through penetration of the organ by adjacent caseating abdominal lymph nodes (5).

In most cases, pancreatic and peripancreatic tuberculosis evolves through multiorgan damage; however, primitive forms exist and are isolated in $25 \%$ of cases (6).

In the present case, the initial diagnosis was a locally advanced pancreatic tumour and EUS-guided FNA helped to rectify the diagnosis.

The clinical significance of this observation is based, in part, on the rarity of pancreatic tuberculosis in France and on the significant value of EUS-guided FNA in the diagnosis. This is rare enough (7) to

${ }^{1}$ Department of Gastroenterology, Strasbourg University, Strasbourg; ${ }^{2}$ Department of Hematology, University of Nice, Nice, France

Correspondence: Dr Adrien Sportes, Department of Gastroenterology, Strasbourg University, 1 place de l'hôpital, 67000 Strasbourg, France.

Telephone 0-33-369-55-1008, fax 0-336-955-0315, e-mail adrien.sportes@chru-strasbourg.fr

Received for publication March 14, 2013. Accepted May 6, 2013 


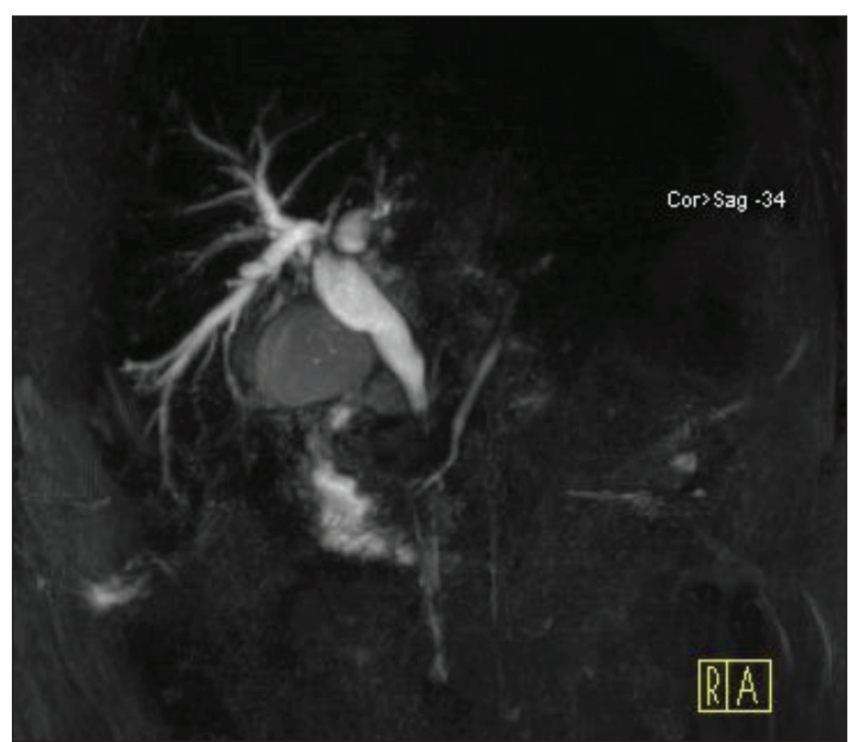

Figure 2) Magnetic resonance cholangiopancreatography showing dilation of intrahepatic and extrahepatic bile ducts upstream of a pancreatic mass

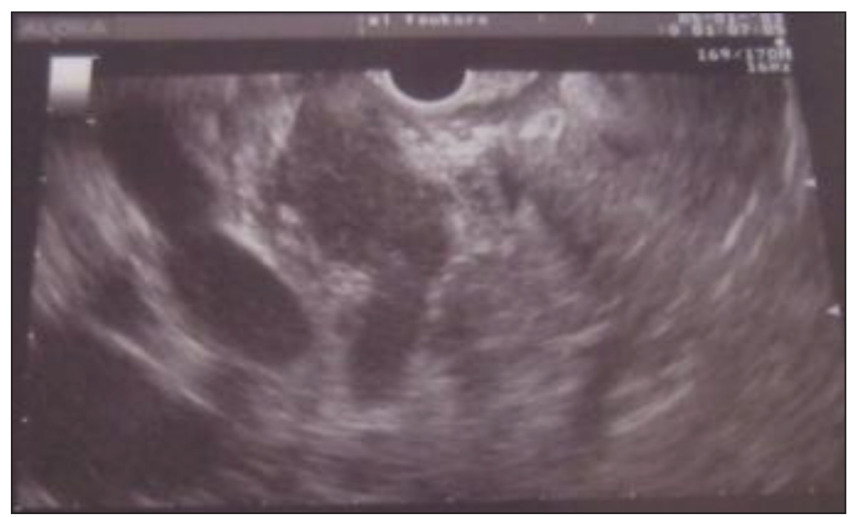

Figure 3) Endoscopic ultrasonography showing a large tumour of the pancreatic head region compressing the common bile duct

be reported because the diagnosis is most often made intraoperatively during exploratory laparotomy (8).

A retrospective study involving patients with pancreatic tuberculosis (9) reported the sensitivity of EUS-FNA to be $76 \%$, without major complications. These data are comparable with those found in pancreatic cancers (10). Therefore, surgical management is not indicated as first-line management.

In our patient, the presence of jaundice was indicative of pancreatic tuberculosis. This mode of revelation is rare, although similar cases are found in the literature $(11,12)$.

The usual clinical manifestations are chronic abdominal pain, pancreatic mass suggestive of cancer (13-15), obstructive jaundice (13), gastrointestinal bleeding (16), acute or chronic pancreatitis (17), or pancreatic abscess (18). However, signs of tuberculous infection may be absent (19).

This disease is usually found in patients in highly specific situations (eg, residents in endemic areas, immunocompromised individuals). A diagnosis of pancreatic tuberculosis should be considered in a pancreatic mass. This diagnosis is a challenge due the rarity of the disease itself and its insidious presentation of combined nonspecific signs and symptoms or, conversely, mimicking the clinical presentation of pancreatic carcinoma, as observed in our patient.

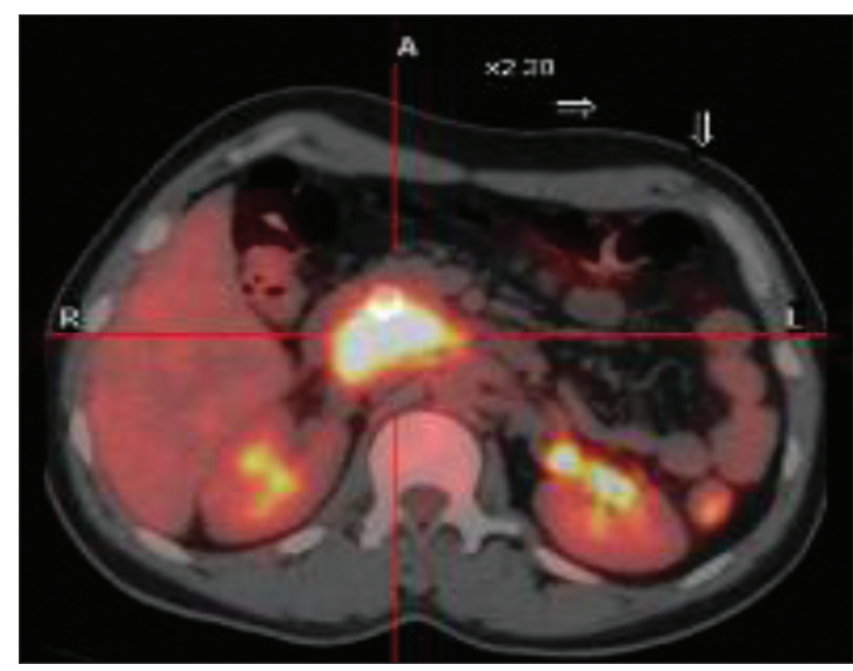

Figure 4) Positron emission computed tomography scan showing intense hypermetabolism characterizing the large swelling of the pancreatic head

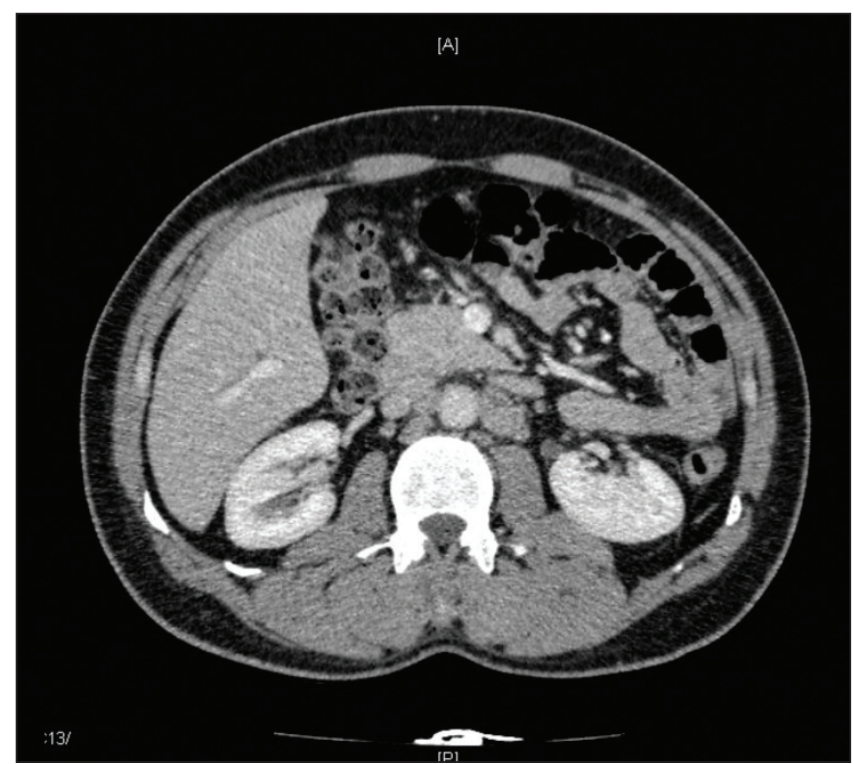

Figure 5) Abdominal computed tomography showing decreased tumour mass after quadruple antibiotics over a six-month period

The increasing incidence of pancreatic tuberculosis is due, in part, to disease expansion brought on by increasing immigration from endemic countries to countries in which detection and treatment is more effective and widely available (20).

\section{CONCLUSION}

A diagnosis of pancreatic tuberculosis is rare and is difficult by clinical presentation alone, which can sometimes be misleading. It is part of the differential diagnosis of a pancreatic tumour, especially in migrant patients from endemic countries. Endoscopic biopsy is necessary to establish a diagnosis and avoid laparotomy. This diagnosis should not be ignored and, with the appropriate therapy, tuberculosis can be cured.

DISCLOSURES: The authors have no financial disclosures or conflicts of interest to declare. 


\section{REFERENCES}

1. Lo SF, Ahchong AK, Tang CN, Yip AW. Pancreatic tuberculosis: Case reports and review of the literature. J R Coll Surg Edinb 1998;43:65-8

2. Auerbach O. Acute generalized miliary tuberculosis. Am J Pathol 1944:20:121-36.

3. Paraf A. Menager C. Texier J. Tuberculosis of the pancreas and tuberculosis of the lymph nodes of the upper region of the abdomen. Rev Med Chir Mal Foie 1966;41:101-26.

4. Franco-Parades C, Leonard M, Jurado R, Blumberg HM, Smith RM. Tuberculosis of the pancreas: Report of two cases and review of the literature. Am J Med Sci 2002;323:54-8.

5. Stock KP, Riemann JF, Stadler W Rosch W. Tuberculosis of the pancreas. Endoscopy 1981;13:178-80.

6. Fischer G, Spengler U, Neubrand M, Sauerbruch T. Isolated tuberculosis of the pancreas masquerading as a pancreatic mass. Am J Gastroenterol 1995;90:227-30.

7. Borentain P. Pancreatic tuberculosis: An unusual cause of obstructive jaundice accessible to endoscopic management Gastroenterol Clin Biol 2007;31:1024-7.

8. Beaulieu S. Pancreatic tuberculosis: A rare cause of pseudoneoplastic obstructive jaundice. Gastroenterol Clin Biol 2004;28:295-8.

9. Song TJ, Lee SS, Park do H, et al. Yield of EUS-guided FNA on the diagnosis of pancreatic/peripancreatic tuberculosis. Gastrointest Endosc 2009;69:484-91.

10. Chang KJ. The clinical utility of endoscopic ultrasound-guided fineneedle aspiration in the diagnosis and staging of pancreatic carcinoma. Gastrointest Endosc 1997;45:387-93.
11. Crowson MC, Perry M, Burden E. Tuberculosis of the pancreas: A rare cause of obstructive jaundice. Br J Surg 1984;71:239.

12. Chen CH, Yang CC, Yeh YH. Yang JC. Chou DA. Pancreatic tuberculosis with obstructive jaundice a case report. Am J Gastroenterol 1999;94:2534-6.

13. Shan YS, Sy ED, Lin PW. Surgical resection of isolated pancreatic tuberculosis presenting as obstructive jaundice. Pancreas 2000;21:100-1.

14. El Mansari O. Pancreatic tuberculosis: Report of two cases. Gastroenterol Clin Biol 2003;27:548-50.

15. Rezeig MA, Fashir BM, Al Suhaibani H, Al Fadda M, Amin T, Eisa H. Pancreatic tuberculosis mimicking pancreatic carcinoma: Four case reports and review of the literature. Dig Dis Sci 1998;43:329-31.

16. Brusko G, Melvin WS, Fromkes JJ, Ellison EC. Pancreatic tuberculosis. Am Surg 1995;61:513-5.

17. Desai SR, Bhanthunmavin K, Hollands M. Primary pancreatic tuberculosis: Presentation and diagnosis. Aust N Z J Surg 2000;70:141-3.

18. Park AJ. Pancreatic tubercular abscess. Pancreas 1993;8:137-8.

19. Takhtani D, Gupta S, Suman K, et al. Radiology of pancreatic tuberculosis: A report of three cases. Am J Gastroenterol 1996;91:1832-4.

20. Woodfield JC, Windsor JA, Godfrey CC, Orr DA, Officer NM. Diagnosis and management of isolated pancreatic tuberculosis: Recent experience and literature review. ANZ J Surg 2004;74:368-71.

The Canadian Journal of Gastroenterology is considering a limited number of submissions for IMAGE OF THE MONTH. These are based on endoscopic, histological, radiological and/or patient images, which must be anonymous with no identifying features visible. The patient must consent to publication and the consent must be submitted with the manuscript. All manuscripts should be practical and relevant to clinical practice, and not simply a case report of an esoteric condition. The text should be brief, structured as CASE PRESENTATION and DISCUSSION, and not more than 700 words in length. A maximum of three images can be submitted and the number of references should not exceed five. The submission may be edited by our editorial team. 


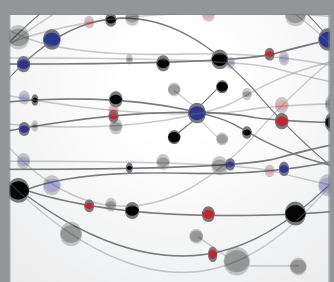

The Scientific World Journal
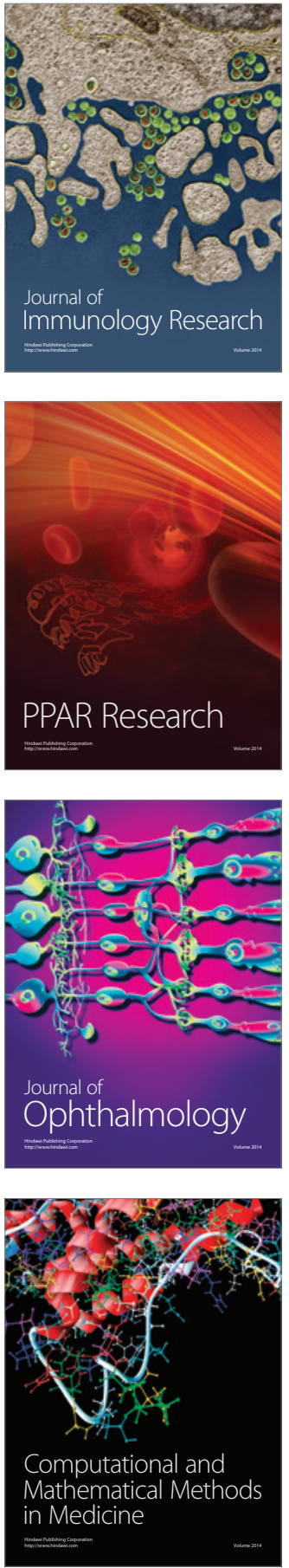

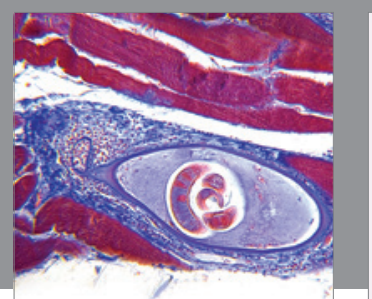

Gastroenterology Research and Practice

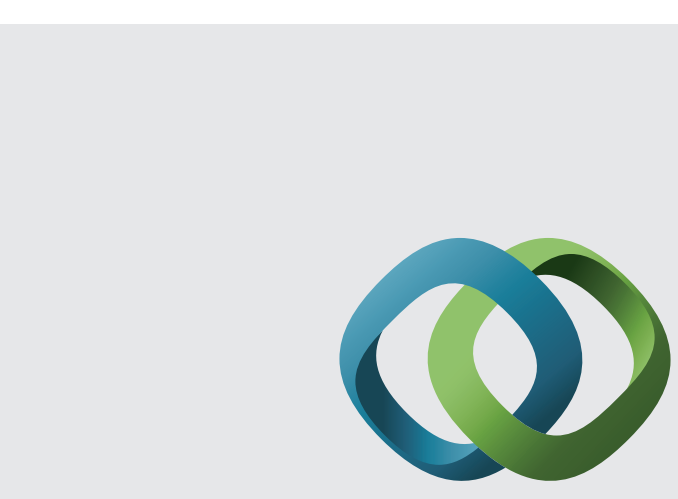

\section{Hindawi}

Submit your manuscripts at

http://www.hindawi.com
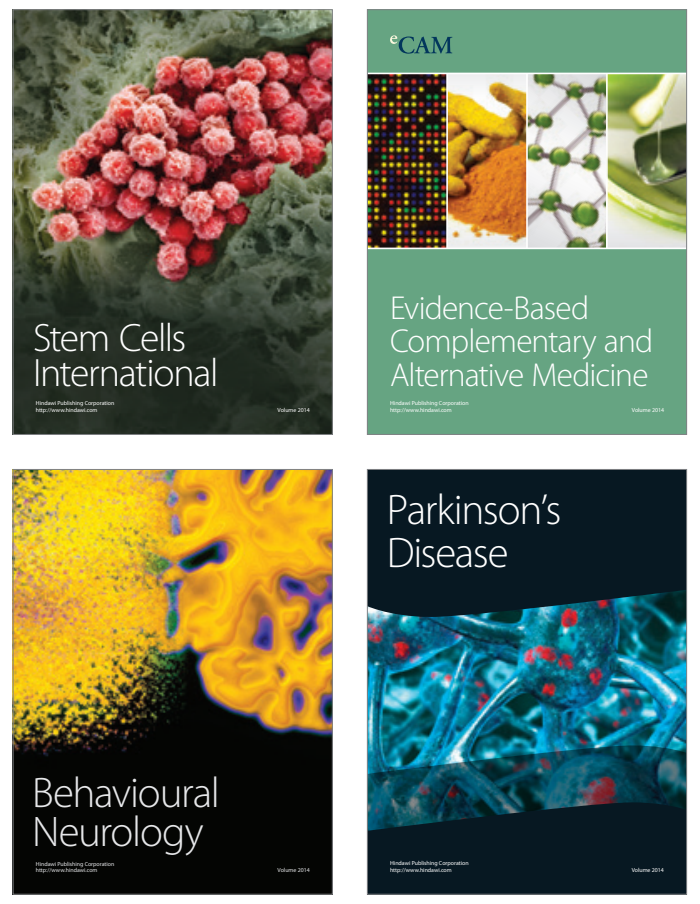
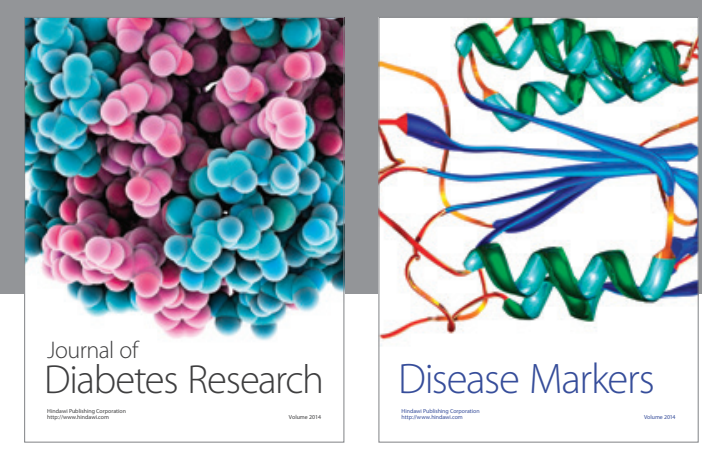

Disease Markers
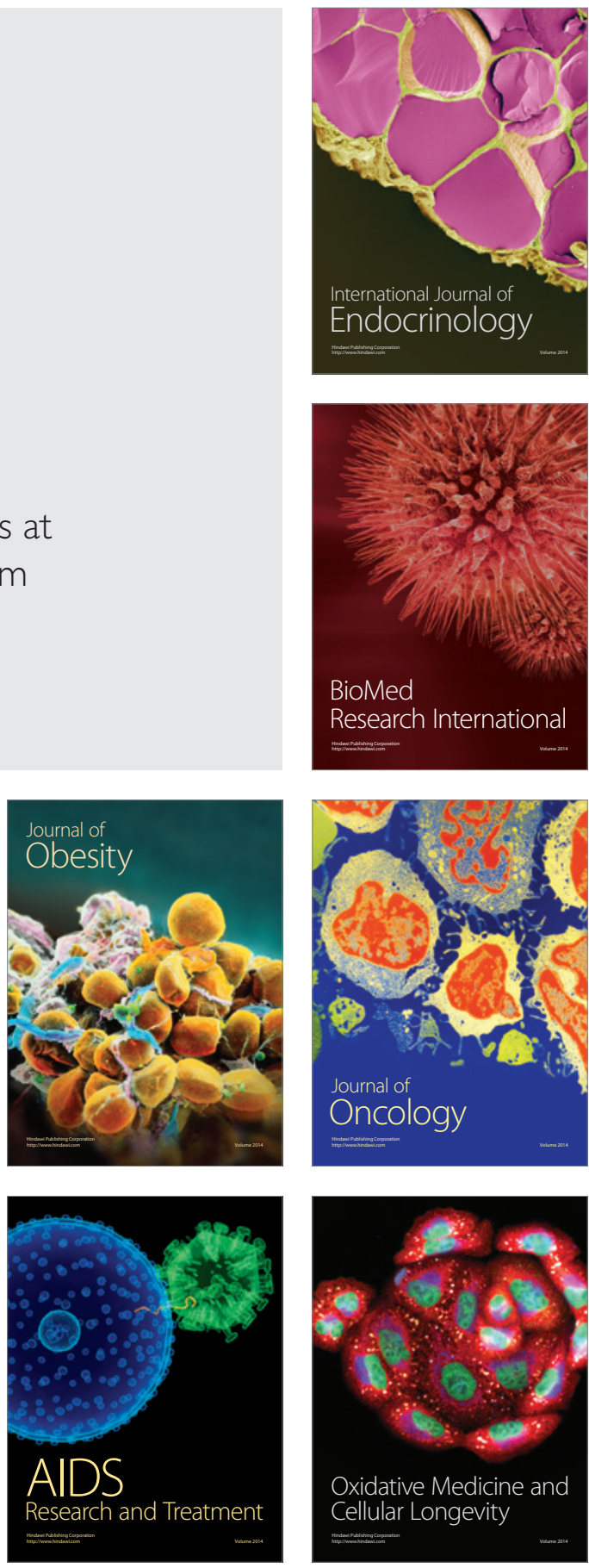